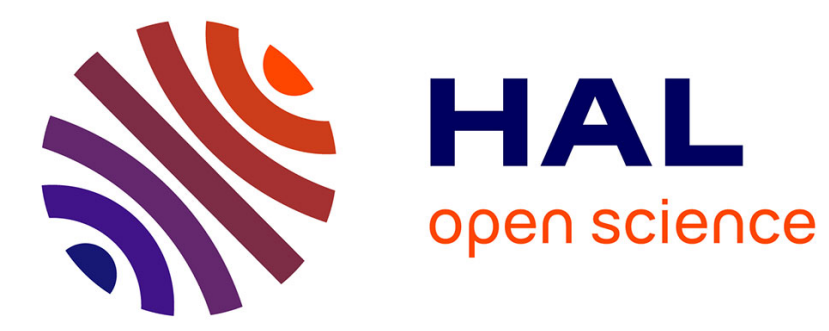

\title{
Establishing bioequivalence of veterinary premixes (Type A medicated articles)
}

\author{
Robert Hunter, Peter Lees, Didier Concordet, Pierre-Louis Toutain
}

\section{To cite this version:}

Robert Hunter, Peter Lees, Didier Concordet, Pierre-Louis Toutain. Establishing bioequivalence of veterinary premixes (Type A medicated articles). Journal of Veterinary Pharmacology and Therapeutics, 2012, 35 (1), pp.53-63. 10.1111/j.1365-2885.2012.01368.x . hal-01191254

\section{HAL Id: hal-01191254 \\ https://hal.science/hal-01191254}

Submitted on 22 Jan 2016

HAL is a multi-disciplinary open access archive for the deposit and dissemination of scientific research documents, whether they are published or not. The documents may come from teaching and research institutions in France or abroad, or from public or private research centers.
L'archive ouverte pluridisciplinaire HAL, est destinée au dépôt et à la diffusion de documents scientifiques de niveau recherche, publiés ou non, émanant des établissements d'enseignement et de recherche français ou étrangers, des laboratoires publics ou privés. 


\section{Establishing bioequivalence of veterinary premixes (Type A medicated articles)}

\section{R. P. HUNTER* \\ P. LEES ${ }^{\dagger}$ \\ D. CONCORDET \& \\ P.-L. TOUTAIN}

*Elanco Animal Health, Greenfield, IN, USA; ${ }^{\dagger}$ Royal Veterinary College, Hatfield, Hertfordshire, UK; ${ }^{\dagger} U M R 1331$ Toxalim, INRA, Université de Toulouse, INPT, ENVT, Toulouse Cedex 03, France
Hunter, R. P., Lees, P., Concordet, D., Toutain, P.-L. Establishing bioequivalence of veterinary premixes (Type A medicated articles). J. vet. Pharmacol. Therap. 35 (Suppl. 1), 53-63.

a) Key issues concerning Premix (Type A medicated articles) Bioequivalence evaluations:

1) This is a complex issue concerning both route of administration and formulation.

2) If the animal is not at the bunk/trough, the animal is not selfadministering (eating medicated feed), thus there can be no drug absorption.

b) Differing opinions among scientists and regulatory authorities/expert bodies regarding:

1) No harmonization on how to design, conduct, and interpret in vivo studies.

2) Applicability of biowaivers to Type A (premix) products.

3) Why are topdress and complete feed considered differently? Are they different formulations or different routes of administration?

4) Single dose vs. multi-dose studies.

5) What is the final formulation?

c) What are the next steps:

1) Harmonize current bioequivalence guidelines through the VICH process.

2) Determine the applicability/non-applicability of the Biopharmaceutical Classification System (BCS).

3) Establish the Total Mixed Ration (i.e. formulation) effects.

4) Define the test subject (individual, pen, etc.).

(Paper received 15 December 2011; accepted for publication 16 December 2011)

R. P. Hunter, Elanco Animal Health, 2500 Innovation Way, Greenfield, IN, USA. E-mail: hunterrp@lilly.com

\section{INTRODUCTION}

Premixes (Type A medicated articles) are veterinary dosage forms, just as are solutions, suspensions, tablets, capsules, pastes, pour-ons, etc. Therefore, in developing a new premix product, a manufacturer will be expected to support medicinal claims by the provision of appropriate data on quality, safety and efficacy. As well as efficacy in clinical trials, it will be normal procedure to provide pre-clinical data on pharmacological properties together with the pharmacokinetic profile of the active constituent(s). Premixes are not aqueous solutions and they are not formulated for intravenous administration, so that exclusions from in vivo testing of a generic premix formulation may not apply in all cases. For generic premix formulations, it is just as essential that bioequivalence to a pioneer product (with established efficacy and safety) based on detailed pre-clinical and clinical assessments is established, as for other veterinary medicinal product formulations. There is, however, the possibility of granting waivers from in vivo studies for premix formulations; this is a subject on which there is no general agreement nor internationally harmonized set of guidelines. Each regulatory authority issues separate guidance. The key considerations therefore are: (i) can premixes be eligible for consideration of a biowaiver; (ii) if biowaivers can be granted, what are the criteria; (iii) how do dissolution testing methods fit within the criteria; (iv) what is the role of the Biopharmaceutical Classification System (BCS) in the evaluation of premix biowaivers.

One of the pivotal assumptions of bioequivalence is that, if two concentration profiles are the same, so are the pharmacological/therapeutic/safety effects. Therefore, bioequivalence trials 
aim at establishing whether or not the concentration-time profiles obtained with two formulations are similar. For a drug given by a standard extravascular route, e.g. tablets administered orally, the essential comparison of two concentration profiles can be achieved by comparing their AUC and $C_{\max }$ values. Thus, we conclude that two formulations are bioequivalent when their AUC and $C_{\max }$ are similar within predefined Confidence Intervals. Whilst establishing bioequivalence for generic premixes may present particular challenges, these cannot constitute a logical basis for abandoning or lessening bioequivalence requirements. Proposals, either to accept no requirements or to adopt lower than generally accepted standards for bioequivalence determination, cannot on efficacy and target species safety grounds be acceptable. There are also considerations of human food safety. Moreover, the fact that most premixes contain as the sole or main active constituent a drug of the antimicrobial, anthelmintic, or anticoccidial classes raises the additional consideration of resistance, which does not apply to other drug classes in the veterinary armamentarium. Thus, provided that the pioneer premix is labelled at a dietary concentration that reaches minimally effective systemic exposure values in the targeted patient population, a generic premix formulation which provides lower drug concentrations in biological fluids and tissues or a generic premix that has greater variability in systemic exposure when compared to the pioneer formulation runs the potential risk not only of reduced efficacy but also increased emergence of resistance to that drug. It is widely recognised that administered dose and hence resulting blood and biophase (or tissue biophase) concentrations are the important determinants of resistance emergence and therapeutic efficacy. Therefore, the potential for the emergence of antimicrobial resistance is an added concern, impinging on both efficacy and safety.

\section{PREMIXES AND FINAL PRODUCTS}

Premixes are veterinary formulations, designed for the prophylaxis, metaphylaxis (control), or therapy of microbial, anthelmintic or coccidial diseases, or for the administration of non-antimicrobial growth promotion compounds by addition to animal feed. Like water soluble products formulated for addition to drinking water, feed containing premix is: (i) normally presented to groups of animals and; (ii) consumed intermittently over a period of time. This raises unique and challenging considerations in relation to the conduct of in vivo studies to establish bioequivalence of generic formulations.

A typical premix might contain $10 \% \mathrm{w} / \mathrm{w}$ active substance together with $90 \% \mathrm{w} / \mathrm{w}$ excipients. The incorporation rate into final feed at $99.5 \% \mathrm{w} / \mathrm{w}$ of feed plus $0.5 \% \mathrm{w} / \mathrm{w}$ of premix will give a final concentration of $0.05 \% \mathrm{w} / \mathrm{w}$ active substance. From a therapeutic point of view, the feed plus premix should be considered to be the final dosage form, but from a regulatory point of view this final dosage form is not the approved dosage form as the premix itself is the approved medicinal product or formulation. The feed and premix contains the active drug substance in a lower concentration than is achieved with many other veterinary medicinal dosage forms (vide infra), often shows lower bioavailability and may be labelled at lower doses than its parenteral counterpart. It should also be noted that the active drug substance in the premix is often a granulated product containing fermentation biomass and/or excipients, and it is subject to a manufacturing process.

Thus, premixes comprise drug substance of a particular character (which may vary in relation to key properties such as particle size, crystal structure etc.) and at a given strength, combined with excipients. Given the nature of the dosage form, differences in manufacturing process and the potential differences in drug concentration, particle size and nature and concentrations of excipients between a pioneer and generic premix product, it seems logical to conclude that there can be no scientific rationale for assuming bioequivalence without recourse to in vivo studies, at least in some circumstances.

These factors were considered at the 2001 meeting of the American Academy of Veterinary Pharmacology and Therapeutics and reported by Martinez et al. (2002a). It was agreed that drug administered in feed presented a unique challenge in the assessment of bioequivalence. Participants were asked to explore the question of whether medicated premixes should be granted biowaivers, regardless of whether or not there are differences in 'inactive' ingredients. Delegates expressed concern over the automatic granting of biowaivers, because of issues such as particle size, polymorphism and excipient effects. Excipients and manufacturing processes were considered to impact potentially not only on drug dissolution but also on uniformity of drug dispersion within the feed. While in the US and EU the manufacturing of Type A medicated articles and their corresponding ability to be formulated into medicated feed is reviewed and evaluated in the same way, independently of the manufacturer (i.e. the Chemistry Manufacturing and Controls), this is not the case in many other regions of the world, as segregation, homogeneity and other feed mixing requirements are neither evaluated nor required for a generic premix. Moreover, this group pointed out that in vitro dissolution testing would be difficult, though not impossible, to conduct because of the clumping behaviour of food substances (Martinez et al., 2002a).

For premixes, the dosage form for which bioequivalence must be established (in the final feed) comprises as little as one part in 2000 of active substance in a carrier composed of minerals and vitamins, but which consists for much the greatest part of vegetable matter from various sources e.g. corn, wheat, soybean, etc. Moreover, the feed into which premix is incorporated will vary, so that the final 'product' is in fact a series of products, each with its own relative bioavailability. Hence, it would be entirely possible for bioequivalence to be demonstrated (or not) between a given generic premix and a pioneer product, depending on its interaction with the particular feed selected for use in the bioequivalence study. Moreover, the reference product itself, when incorporated into two feeds of differing composition, may create two formulations which are not bioequivalent and this is a possible issue which must be dealt with in designing bioequivalence studies for premixes; in this 
situation, if the two tested formulations are equally influenced by the food, they can nevertheless be bioequivalent; in contrast if there is a differential food effect (i.e. a food $\times$ formulation interaction effect) the two formulations might be bioequivalent for one type of food but not for all types of food. Few examples of such interactions have been published (Shryock et al., 1994) but data were reported in man for ciprofloxacin (Neuhofel et al., 2002); it has also been observed for modified-release products (Schug et al., 2002a) and it was quoted that the type of formulation-dependent food effect was often not predictable from the in vitro characteristics of the dosage form (Schug et al., 2002b).

\section{INFLUENCE OF FEED ON DRUG BIOAVAILABILITY}

There are innumerable cases, cited in the veterinary literature, of influence of feeding stuff on rate and extent of absorption of active constituents from orally administered products. Both the granules (in terms of size, texture, and applied compression force) and the excipients may differ between the pioneer and generic products. Another issue is stability. An example is the aminopenicillins, ampicillin and amoxicillin. These are not stable at high temperatures and in some final formulations manufactured at a high temperature the active ingredient concentration in the final product could be less than the nominal concentration. Of interest in relation to premixes are those cases, in which relative bioavailability has been modified by vegetable matter feedstuffs. Two examples will suffice to illustrate this point. Several drug classes [the NSAIDs, phenylbutazone, meclofenamate, and flunixin in Lees' laboratory (Maitho et al., 1986; Lees et al., 1988)] and sulphonamides and trimethoprim in the studies of Van Duijkeren et al. (1996) have been shown to bind to components of vegetable feedstuffs. This was demonstrated in in vitro spiking experiments with NSAIDs conducted in buffers of varying $\mathrm{pH}$. The degree of binding to feedstuffs varied with conditions, including $\mathrm{pH}$, drug concentration, temperature, duration of exposure etc. For some NSAIDs e.g. phenylbutazone and meclofenamate, in vitro binding to hay and pony nuts was high $(>98 \%)$ and binding to digesta in vivo was also demonstrated (Lees et al., 1988). In other in vivo studies it was shown that binding to feed and digesta influenced the pattern of absorption of phenylbutazone and flunixin and was postulated to be the cause of: (i) delayed absorption; and (ii) two or more peaks in the plasma concentration-time profiles (Maitho et al., 1986; Welsh et al., 1992).

Similar binding of drugs in premixes to components of vegetable feed will potentially alter both rate and extent of absorption of drug from the in-feed formulation, for example by affecting the dissolution rate of drug in vivo in the gastrointestinal tract. This has been demonstrated for ivermectin in sheep (Ali \& Hennessy, 1996). The implication for in vivo bioequivalence trials is that binding to digesta is one factor which may increase the inter-, or/and the intra-animal and especially the inter-occasion variability in rate and extent of absorption and thus potentially reduce the likelihood of concluding bioequivalence.

Some drug substances, even under conditions which are optimal for absorption after oral dosing, have a low bioavailability. In addition, as a result of sequestration within and possible in vivo binding to digesta after administration, the bioavailability may be further decreased, impacting on attempts to demonstrate the bioequivalence of premix formulations by in vivo studies. An example is tylosin, for which bioavailability is $<10 \%$ in pigs and chickens. Consequently, the bioequivalence considerations that apply in general to highly variable drugs may specifically apply to some premix formulations. As discussed by Toutain and Bousquet-Melou (2004), variability (expressed as $\mathrm{CV} \%$ ) is almost invariably high when absolute bioavailability is low. Therefore, the approaches to bioequivalence for highly variable drugs proposed by Haidar et al. (2008a,b) and Davit et al. (2008) may be applicable to some premixes. This approach is based on scaling of an average bioequivalence criterion to the within-subject variability of the reference product in a cross-over study, together with a point estimate constraint imposed on the geometric mean ratio between the test and reference products. The method requires replication of the reference product treatment in each individual. It involves use of a three sequence, three period, two treatment study design. If a cross-over design is not possible, this approach is not applicable.

\section{STEPS TO MINIMISE SOURCES OF VARIABILITY IN IN VIVO STUDIES}

As with all in vivo bioequivalence studies, all possible steps should be taken, in bioequivalence trials of premixes, to minimise sources of variability, other than those relating to the two formulations being compared. Bioequivalence studies normally involve using maximum recommended dose with single administration as a tablet, capsule, injection, etc. at a fixed time. For premixes, however, it is inherent in the nature of the product that dosing is not undertaken at a fixed time. The medicated feed is, rather, presented at a given instant in time and, even if nonmedicated feed has been withheld for a fixed period before presenting medicated feed, the medicated feed will be consumed over a period of time and is likely to be discontinuous. These factors will vary between animals. Moreover, in clinical use, the premix admixed with feed is presented on a group basis. Actual dosage consumed will therefore vary between animals in the pen and be dependent upon both behaviour of the individual within the group and the health status of an individual. The word individual is not exchangeable with animals; it is a 'generic' word from L. individuus meaning 'indivisible'. Current average bioequivalence trials require this property to hold in a 'virtual animal': the 'average individual'. For this individual to be precisely qualified, it is necessary to define the population from which this average individual is obtained. In current regulatory guidelines, the population is implicitly assumed to be homogeneous with no specific strata among which the pharmacokinetics 
may differ. In this case, a simple random sampling of individuals allows the population average to be well estimated.

A key question is, should the manner of clinical use in relation to feeding regimen be reflected in the bioequivalence trial design. For premixes, should the presentation of feed simulate that of clinical use to groups or should the weighed feed be presented on an individual animal basis? It is commonly acknowledged that food intakes of animals in a given pen are not independent of each other: the food intake of an animal fed alone (e.g. in a metabolic cage) is not the same as when the animals are collectively fed. As the animal feed intakes are not the same, it is expected that the individual pharmacokinetic profiles would differ. Another consideration in study design is that the removal of an animal from a pen, in order to collect a blood sample, involves an unavoidable disruption to the eating schedule, and this could have a significant influence on the plasma concentration-time profile, thereby impacting on bioequivalence determination. It is neither practicable nor relevant to dose animals with the in feed dosage form as a bolus at a single time-point. If the premix itself, not admixed with feed, was administered orally as a bolus to unfed animals at a fixed time, this would differ from the circumstances of clinical use.

The above considerations present significant challenges to study design to evaluate bioequivalence between generic and pioneer premixes, when formulated into the final in-feed dosage form. However, these considerations are not unique to premixes. A small tablet or a potent drug may be admixed into a large feed for oral dosing. The final 'dosage form' may comprise $<1 \%$ of tablet and the tablet might contain as little as $0.5 \% \mathrm{w} / \mathrm{w}$ active constituent, so there might be as little as one part in 300000 active constituent in the feed. Moreover, it is entirely possible that two tablet formulations might be shown to be bioequivalent with a particular (e.g. low fat) feed but not when admixed with another (e.g. high fat) feed. Factors such as age and breed may lead to differences in gastrointestinal tract physiology, thereby impacting, selectively, on the formulation's rate and extent of drug absorption. Therefore, the principles which underlie the requirement for conduct of bioequivalence studies for generic tablet dosage forms are similar in some respects to those which apply to premixes.

Once the population is clearly defined, as a collection of farms or a collection of pens, several proposals for bioequivalence definitions can be made: (1) the premix formulations should give similar exposures in an 'average hypothetical farms/pens'; (2) the statistical distribution of the drug exposures of the two formulations among breeders/pens should be close; (3) for most farms/pens, the formulations should give similar exposures. Considerations 1,2 , and 3 , respectively, lead to average, population and individual bioequivalence trials with the farm/pen as the 'individual'. It is now relevant to consider what the different kinds of bioequivalence imply.

If the farm is the individual, two premix formulations would not be bioequivalent if there is some farm-specific factor able to interact with the formulation. Such factors could be the type of food, the way the premix is mixed with the food etc. If the pen is the 'individual', two premix formulations would not be bio- equivalent if some pen-specific factor interacts with the formulation. When the two formulations are declared to meet average bioequivalence criteria, the population means of their exposures (AUC and $C_{\max }$ ) are similar. In this definition, only the representativeness of the 'individuals' is important. It can be ensured by randomly sampling these 'individuals' within the population. As long as the sample is representative, sources of variation in pharmacokinetic profiles cannot bias the estimation of population means. Therefore, they can be regarded as noise, because they render imprecise the estimation of the population means. Consequently, the study can be organized so that it minimizes this variability factor.

\section{CHOICE OF ANALYTICAL METHOD AND SAMPLE MATRIX FOR EVALUATING BIOEQUIVALENCE OF PREMIXES IN VIVO}

Measurement of plasma concentration-time profiles might not be possible for all premix generic products or in all circumstances. For example, absolute bioavailability may be low (as for example monensin, which is rapidly metabolised in the liver). Hence, concentrations in plasma are below the lower limit of quantitation (BLOQ) of the analytical method for all samples. Alternatively, concentrations of drug might be BLOQ because of high drug potency. Recent advances in LC/MS/MS analytical methods will not always address this problem. Nevertheless, lack of a sufficiently sensitive method should not be the sole reason for not conducting a blood level bioequivalence study.

In practice, for various reasons, none of the alternatives to monitoring plasma drug concentration are likely to be applicable for premixes for evaluating the bioequivalence of generic and pioneer formulations. Therefore, it may be more appropriate to conduct, as an alternative approach, a clinical end-point bioequivalence study to support bioequivalence instead of a blood level study. It must be recognised that this will increase the number of animals involved and the study cost, but it is likely to be a realistic alternative to the use of the conventional plasma concentration-time profile approach to bioequivalence determination. However, this raises other questions, such as what confidence intervals should be used in a pharmacodynamic or clinical endpoint bioequivalence study?

\section{DESIGN AND CONDUCT OF IN VIVO BIOEQUIVALENCE STUDIES FOR PREMIXES}

Particular considerations relating to study design are as follows:

a) The final feed into which a premix is incorporated may influence rate and extent of absorption. However, this alone does not provide a scientific basis for a waiver of the need to conduct a bioequivalence study, because the circumstance does not differ in principle from one in which a tablet is administered in either the fed or fasted state to a companion animal, where the meal composition is likely to impact on 
absorption rate and extent. Therefore, the selection of a commonly used feed for both pioneer and generic premixes will give a reasonable assurance of bioequivalence of the two products.

b) In general, single dose studies are preferred, by regulatory authorities, to multiple dose studies to evaluate two products for bioequivalence, as the rising phase of the plasma concentration-time curve will commonly better define absorption rate (as indicated by $C_{\max }$ ) than when a multiple dose study (with the objective of achieving steady state) is used. However, final formulations based on premixes are given as multiple doses presented in feed. Moreover, they are presented for consumption at a given time but actual ingestion is discontinuous and spread over a period of time and both of these factors may increase interanimal variation in $C_{\text {max }}$. These factors and drug binding (e.g. sorption/desorption processes) to digesta may lead to multiple peaks in plasma concentration. In addition, as discussed above, variability is likely to be increased when animals are removed from feed in order to collect blood samples; this also can have a significant effect on $C_{\max }$ (Toutain \& Bousquet-Melou, 2004). For AUC, two bioequivalence variables should be selected, each based on values over the time interval between presentation of the first and the final feeds $\left(\mathrm{AUC}_{0-\tau}\right)$, where the second time interval corresponds to pharmacokinetic steady state. Any influence of the feed will be magnified after multiple doses and reflected in the variable $\mathrm{AUC}_{0-\tau}$ at steady state. Another possibility would be to conduct Monte Carlo simulations, using them to explore the potential for alternative study designs. These simulations, with the correct assumptions, would be likely to provide significant information on the type and duration of sampling and correct selection of pivotal pharmacokinetic parameters for analysis.

c) To date, regulatory authorities have accepted the demonstration of average bioequivalence, as providing sufficient assurance on essential similarity to conclude therapeutic equivalence. However, when group medication is used, population bioequivalence documenting the variance as well as the mean of the performances of the two tested formulations is likely to be more appropriate. Premixes are almost invariably administered to groups of animals. Hence, it is possible that an interaction exists between behavioural patterns (including food intake) and formulations, leading to identical average bioequivalence but to very different interindividual variability for the two formulations. Such variability could be critical in terms of emergence of resistance to antimicrobial drugs.

In light of these considerations, an approach to in vivo testing of bioequivalence could be both a gavage study and a feed consumption study. The former would provide information to confirm similar plasma levels across formulations. The latter would confirm that consumption of the medicated feed is the same across the products.

\section{EXEMPTIONS FROM IN VIVO BIOEQUIVALENCE STUDIES FOR PREMIXES}

In 2000, the document 'Waiver of in vivo bioavailability and bioequivalence studies for immediate-release solid oral dosage forms', based on a Biopharmaceutics Classification System (BCS), was issued jointly on behalf of the U.S. Department of Health and Human Services, the Food and Drug Administration and the Center for Drug Evaluation and Research (CDER). It was based on publications in the scientific literature by Amidon et al. (1995) and Yu et al. (2002). The basis of the classification is recognition that drug dissolution and gastrointestinal permeability are the fundamental parameters controlling rate and extent of absorption. This document was designed specifically in relation to oral products intended for human use and is therefore based on considerations of (i) immediate release solid oral dosage forms (IRSODs) e.g. tablets, capsules used in humans and (ii) the anatomy and physiology of absorption from the human gastrointestinal tract after oral dosing. Therefore, the guidance cannot be applied without modification: (i) to animals with significantly different anatomical and physiological absorptive systems (e.g. poultry and cattle) to humans; or (ii) to premixes which differ significantly from formulations used in humans. Nevertheless, the extent to which these considerations might be adapted to provide biowaivers for veterinary premixes has been published (CVM/FDA Guidance for Industry \#171).

The BCS system constitutes a scientific framework for classifying drug substances into four groups based on two factors, namely aqueous solubility and intestinal permeability:

Class I: High Solubility - High Permeability: when formulated as immediate release products dissolution rate exceeds gastric emptying time and bioavailability is expected to approach $100 \%$. In vivo bioequivalence data should not be necessary to ensure product comparability i.e. biowaiver normally granted.

Class II: Low Solubility - High Permeability: these compounds are likely to be dissolution rate limited and a correlation between in vivo bioavailability and in vitro dissolution rate may be observed.

Class III: High solubility - Low Permeability: dissolution is likely to be rapid but absorption is permeability rate limited. Waiver conditions similar to those for Class I compounds may be appropriate provided test and reference formulations do not contain agents that can modify permeability or gastrointestinal tract transit time.

Class IV: Low solubility - Low Permeability: these compounds tend to be difficult to formulate and may exhibit large interand intra-subject variability in bioavailability but a recent investigation of 124 human bioequivalence studies reported exactly the opposite with the lowest inter-subject variability in AUC for drugs belonging to class IV of the BCS classification and this was explained by an absence of saturation of intestinal transport and enzyme function (Ramirez et al., 2010). 
These two drug substance properties (solubility and permeability) are considered with a third, the dissolution rate of the active constituent from the drug product (which is a formulation property), as the major factors which govern rate and extent of drug absorption. Highly soluble drugs (in the human context) are those for which the highest dose strength is soluble in $250 \mathrm{~mL}$ or less of aqueous medium at three $\mathrm{pH}$ values over the range of 1.0-7.5. In the absence of evidence suggesting instability within the gastrointestinal tract, a drug substance is considered to be highly permeable when human bioavailability is $90 \%$ or greater. A drug product is considered to be rapidly dissolving when not $<85 \%$ of labelled drug substance dissolves within $30 \mathrm{~min}$ under defined conditions. However, the United States Pharmacopeia and European Pharmacopeia tests for dissolution are specifically designed for human solid dosage forms such as tablets and capsules. Whilst premixes may be regarded as solids, they are neither tablets nor capsules and their behaviour in in vitro dissolution tests has been little studied. The use of the BCS, even in human medicine, has also recently been called into question regarding its ability to predict bioequivalence (Ramirez et al., 2010). Ramirez and co-workers report that they were unable, using 124 active substances from all four BCS classes to consistently predict success in the corresponding in vivo bioequivalence study. Compounds in groups BCS 1 and 3 failed in vivo testing in approximately $15 \%$ of studies, with compounds from Class 3 failing in at least $50 \%$ of the studies (Ramirez et al., 2010).

There has been much discussion on the BCS system. Blume and Schug (1999) proposed that Class III compounds may be even better candidates for a biowaiver than Class I drugs, in the absence of excipients which may modify gastrointestinal tract transit time or membrane permeability. This is because bioavailability is not dependent on the release profile of active drug, permeability being the rate limiting process for intestinal absorption. Further support for biowaivers for Class III drugs was provided by a recent workshop (Polli et al., 2008). However, Class III compounds may have site dependent absorption properties, so that transport velocity through the gastrointestinal tract may be strongly affected by excipients such as mannitol which modify gastrointestinal tract transit time. Recently Kortejärvi et al. (2007) carried out pharmacokinetic simulation models to compare $C_{\max }$ and AUC values of IRSODs with oral solutions. The goal was to explore the predictive value of the different classes of the BCS. It was shown that BCS III drugs were less sensitive to the changes in formulation type and gastric emptying rates than BCS I drugs; that most BCS I drugs having elimination rate constants of $<0.2$ per $\mathrm{h}$ showed $<5 \%$ difference in $C_{\max }$ and AUC; that all BCS III drugs are good biowaiver candidates, but half of the BCS I drugs are not; indeed based on their simulations, approximately half of BCS I drugs had a higher risk to fail in bioequivancy than BCS III drugs. For these BCS I compounds, $10-25 \%$ differences in $C_{\max }$ were observed. The remaining BCS I drugs and all BCS III drugs had lower risk to fail, since $<10 \%$ differences in $C_{\max }$ and AUC were observed. It should be noted that these conclusions rely on simulations that are not directly informative for premixes, because only single bolus dose administration was simulated. To document the predictive value of the BCS for premixes, it will be necessary to add a zero-order rate constant to the model to mimic the food intake (i.e. a rather slow arrival of the drug to the stomach) plus a random model (to mimic episodic food intake with its intersubject variability) in order to explore what could be the situation in field conditions for premixes. We conclude that there is a paucity of experimental evidence in veterinary medicine on which to judge definitively the applicability of current BCS approaches and in silico simulations, especially in view of differences in domestic animal physiology. In light of these considerations, we draw attention to the urgent need for experimental data to determine the need, if any, for alterations to regulatory guidelines.

Recently, Benet (2009) reviewed the importance of transporters in determining drug bioavailability in humans. For Class I compounds transporter effects were negligible; for Class III and IV compounds, uptake transporters can be major determinants of bioavailability; for Class II compounds uptake transporters are unimportant but efflux transporters can have major effects on bioavailability. The complexity of issues surrounding the BCS system in relation to biowaivers, is illustrated by the proposal of Benet et al. (2008) 'that regulatory agencies add the extent of drug metabolism (i.e. $\geq 90 \%$ metabolized) as an alternate method for the extent of drug absorption (i.e. $\geq 90 \%$ absorbed) in defining Class I drugs suitable for a waiver of in vivo studies of bioequivalence'. In summary, eligibility for granting a biowaiver should also depend on the extent of pre-systemic (intestinal) metabolism and the likely role of transporters in drug absorption.

Application of the BCS to veterinary pharmaceutical products has been discussed in two major reviews, the first dealing with biopharmaceutics and formulation considerations (Martinez et al., 2002b) and second with physiological considerations (Martinez et al., 2002c). These authors defined the several categories of drug products approved for use in animal feeds as follows:

- 'Type A: these medicated articles are intended solely for use in the manufacture of another Type A, or a Type B or C medicated feed. Type A articles are considered new animal drugs, and may be manufactured with or without carriers (e.g. calcium carbonate, rice hull, and core) and may or may not contain inactive ingredients.

- Type B: these articles are intended solely for the manufacture of another Type B or a Type C medicated feed, and contain a substantial quantity of nutrients (not $<25 \%$ nutritional content, w/w). Type B medicated articles are manufactured by diluting a Type A article or another Type B article. The maximum concentration of $\operatorname{drug}(\mathrm{s})$ in a Type B medicated feed is 200 times the continuous use level for drugs not requiring a withdrawal time, and 100 times the highest continuous use level of disease control and prevention for those compounds associated with a withdrawal time.

- Type C: these articles are intended to be used as the complete animal feed or may be added on the top of the usual ration (i.e. used as a top dress)'. 
Martinez et al. (2002b) and Martinez and Hunter (2010) rightly commented that bioequivalence testing on medicated feeds is extremely complex. Issues to be addressed include: (i) animal variability in feed intake (which will magnify normal pharmacokinetic variation); (ii) palatability; (iii) the question as to whether a change in excipient will have nearly the same impact as that associated with normal fluctuations in the composition and nutrient quality of feed materials; (iv) differences in meal composition; and (v) the uncertainty on methodology for conducting in vitro dissolution studies on medicated articles and/or the final oral dosage form containing granules/pellets. Because, for premixes, the formulation never completely dissolves in vitro, drug is potentially trapped or bound to feedstuff and this may lead to inappropriate conclusions of incomplete in vivo dissolution.

del Castillo and Wolff (2006) have pointed out that granular premixes have become a standard manufacturing practice because of their increased stability and shelf-life and ability to disperse homogeneously in feed. However, the ultimate quality of any premix depends on the rate and extent of active ingredient released in vivo. In this respect not all premixes can be regarded as simple 'pre-solutions'. del Castillo and Wolff (2006) adapted the USP standard dissolution testing method to determine the dissolution in vitro into simulated porcine gastric fluid $(\mathrm{pH}=1.6)$ of four commercial brands of granular chlortetracycline premixes. The test was conducted under standardised conditions with 21 samples collected for analysis over a $120 \mathrm{~min}$ period, to enable determination of the fraction of drug which undergoes dissolution. The results revealed complex patterns of chlortetracycline release and major interactions of the dissolving drug with feed particles. Type of premix was the greatest determinant of rate of drug release, with significant differences reported for the four premixes investigated. In addition, there were major differences in amount of chlortetracycline released over $120 \mathrm{~min}$, as indicated by available-to-total concentration ratios. Approximate percentage ratios were: $45 \%, 51 \%, 64 \%$, and $66 \%$. On average, the chlortetracycline released from the two pioneer premixes was $37 \%$ greater than that from the other two premixes. From these data, the potential to attain bioequivalence in vivo is likely to be achieved only for the two pioneer products. This is not surprising as these products had similar formulations, the principal difference being in the concentration of chlortetracycline in the premix.

del Castillo and Wolff (2006) supplemented the in vitro dissolution data with in vivo pharmacokinetic data, involving administration of chlortetracycline medicated feeds to young pigs (four groups, four animals per group) on an individual animal basis under standardised laboratory conditions. Plasma concentration-time profiles were established for individual animals after the first and again after the seventh medicated meal. They reported the least inter-animal variability in plasma concentration-time profiles for pioneer chlortetracycline and the greatest variation for a generic chlortetracycline formulation. The percentage of time for which plasma concentrations exceeded a breakpoint value of $0.5 \mu \mathrm{g} / \mathrm{mL}$ was determined, as chlortetracycline is classified as a bacteriostatic antimicrobial. There were sub-therapeutic concentrations $(<0.5 \mu \mathrm{g} / \mathrm{mL})$ for up to $14 \mathrm{~h}$ per day for one premix. Finally, it was noted that two premixes were associated, in some pigs, with delayed intake of feed on initial presentation, suggesting the need to take into account palatability when investigating premixes. In summary, these laboratory data on chlortetracycline premix formulations, even if obtained in a limited number of animals and in an experimental setting, have the merit to draw attention to the fact that all premixes for a given active substance cannot be, a priori, assumed to be bioequivalent due to possible product differences in: (i) solubility of the active pharmaceutical ingredient (API); (ii) dissolution rate and extent in vitro; and (iii) not only relative mean bioavailability but also inter-animal variability of pharmacokinetic data in vivo. Properties of chlortetracycline relevant to its absorption rate and extent from any formulation are: (i) a significant likely reduction in bioavailability in the presence of divalent cations (ii) sparing solubility in water and (iii) the requirement to establish solubility in $0.1 \mathrm{~N} \mathrm{HCl}$ and at $\mathrm{pHs} 4.5$ and 7.4, making it unlikely that a biowaiver under U.S. Guidance for Industry 171 would be granted for premixes containing it as the active.

Not all of the API's of each premix can be guaranteed to meet all three BCS criteria, namely highly soluble, rapidly dissolving, and highly permeable (Class I) or two criteria, highly soluble and rapidly dissolving (Class III), thereby potentially allowing the granting of a biowaiver. Data on aqueous solubility will be available for the API of premixes, but new studies may be required to establish solubility at several $\mathrm{pH}$ levels and a fluid volume and composition relevant to conditions within the gastrointestinal tract of each target species. New criteria and guidelines on designing studies for dissolution rates of the active constituents from final medicated feeds should also be required, presumably conducted with a range of feeds of differing composition, under standardised conditions.

Establishing high permeability by in vitro methods alone (e.g. transport rates across Caco-2 cells) may be unsatisfactory for granting a biowaiver. Classification of active constituents into one of Classes I-IV is preferably determined by establishing the absolute bioavailability for the active constituent by: (i) administering the active by two routes, orally as the final formulation and intravenously in a two period, two sequence cross-over design in the target species; or (ii) by mass balance determination (recognising that this method may provide variable estimates). Whilst such a study could be conducted in each target species, manufacturers of generic premixes might be expected to prefer to establish relative bioavailability of their product in comparison with the pioneer product by the classical in vivo bioequivalence route, because such proof could be acceptable for those actives for which species specific absolute bioavailability is $<90 \%$.

In conclusion, accurately predicting absorption rate and extent for active constituents of premixes on the basis of chemical structure, physico-chemical properties (including aqueous solubility and dissolution rates) and permeability will not be appropriate in some instances. As discussed by Burton et al. (2002), drug absorption is a complex process dependent upon drug properties such as solubility and permeability, formulation 
factors, and physiological variables, including regional permeability differences, pH, luminal and mucosal enzymology, and intestinal motility, among others. The many factors influencing rate and extent of absorption of drugs in a veterinary context is the subject of reviews by Martinez et al. $(2002 \mathrm{~b}, \mathrm{c})$.

\section{REGULATORY AUTHORITY GUIDELINES ON GENERIC PREMIXES}

\section{USA}

In the USA general guidelines on bioequivalence are provided in document 35 Bioequivalence Guidance, effective from November 8, 2006. Section II B describes criteria for waiver of requirement to conduct in vivo studies for bioequivalence. The requirement may be waived for 'oral solutions or other solubilised forms'. The criteria are that, in general, the test product is a solution containing the same active and inactive ingredients in the same dosage form and concentration and has the same $\mathrm{pH}$ and physico-chemical characteristics as the reference product. It would therefore seem that oral solutions or solubilised forms, containing either different excipients or the same excipients but in differing concentrations in reference and test products, would not, in general, be eligible for a biowaiver.

Guidelines on Type A Medicated Articles are provided in document 171, Waivers of In Vivo Demonstration of Bioequivalence of Animal Drugs in Soluble Powder Oral Dosage Form Products and Type A Medicated Articles, issued on October 6, 2008. These expand on document 35. Type A medicated articles contain an API with excipients and together they comprise only a small component of the final ingested product. The composition of the final medicated feed varies with the nutritional content of the foodstuffs with which it is mixed. The consideration underlying document 171 is that "with few exceptions, if the drug itself is water soluble across a variety of $\mathrm{pH}$ values, the composition of the Type A medicated article will not affect the bioavailability of the drug in the Type B or Type C medicated feed'. Thus, the assumption is that active ingredient passes rapidly into solution upon contact with fluids of the gastrointestinal tract. Consequently, the rate of absorption is dependent on the rates of gastric emptying and intestinal transit, reflecting only physiological rather than formulation dependent variables. The FDA/CVM recognises an exception 'when the formulation contains substances that could cause pharmacological effects, such as altered gastrointestinal tract transit time, membrane permeability or drug metabolism, or when there is inactivation of the drug by, for example, an excipient that chelates the API'. Thus, CVM 'reserves the right to deny a waiver request if there is any component of the Type A medicated article that is believed to either compromise drug solubility or alter intestinal permeability. Examples of "inactive" ingredients that may be of concern include substances known to alter drug solubilisation (e.g. a chelating agent), intestinal permeability enhancers (e.g. polysorbate 80) or excipients that can alter GI transit time (e.g. osmotically active substances such as sorbitol and mannitol)'. It may be necessary to test experimentally the underlying assumption that water solubility of drug substance is the key or sole consideration. That the composition of the Type A medicated article may also be important in governing dissolution rate, is suggested by the findings of del Castillo and Wolff (2006) for chlortetracycline formulations, as discussed above. In this regard, premixes may differ from simpler formulations, such as capsules and tablets, developed for use in humans.

Whilst feed constituents may affect bioavailability of Type A medicated articles, CVM believes "that this should not be a factor in considering a biowaiver request since the variability in feed constituents between the reference and proposed Type A medicated articles should not be greater than the natural variations that can occur in the final feed to which the animal will be exposed, whether that feed contains the proposed product or the reference product'. For Type A medicated articles containing biomass products, 'generally CVM would deny a biowaiver on the basis of such potential feed ingredient effects only when it has information indicating that specific feed ingredient may have such an effect', that is adverse pharmacological effects or inactivation of active ingredients. It may be necessary to test experimentally the underlying assumption that water solubility of drug substance is the key or sole consideration. That the composition of the Type A medicated article may also be important in governing in dissolution rate, is suggested by the findings of del Castillo and Wolff (2006) for chlortetracycline formulations, as discussed above. In this regard, premixes may differ from simpler formulations, such as capsules and tablets, developed for use in humans.

As drug solubility can be affected by $\mathrm{pH}$ (ionized organic molecules are commonly much more soluble in water than unionized moieties), CVM requires that solubility be tested across a wide range of $\mathrm{pH}$ values, as a condition to grant of a biowaiver. A failure to dissolve in any one $\mathrm{pH}$ condition disqualifies generic products from being a candidate for a biowaiver. However, the CVM biowaiver guidance allows for a dose adjusted definition of solubility based on gastric volume and targeted dose, as dosage will usually be much $<1 \mathrm{~g}$ of drug per $10 \mathrm{~mL}$ of gastric fluid. Thus, the entire dose must dissolve in the gastric volume of the targeted species to avoid the possibility of dissolution-rate limited bioavailability.

The current FDA/CVM opinion is that the criteria for granting of a biowaiver cannot be used to bridge between a Type A medicated article and a water soluble powder. Unlike with tablets, oral boluses, oral suspensions, and injectable formulations, animal behaviour (drinking and eating) determines the actual dose received when the drug is administered in the drinking water or in food. While blood level bioequivalence studies employing gavage dosing may confirm the absence of a formulation effect (which is the sole question applied to products that meet the criteria for approval as an abbreviated NADA), it cannot confirm the comparability of rate and extent of drug intake. This difference in intake may be greater in diseased as compared to healthy animals, since diseased animals tend to go off food before they cease to drink. Therefore, CVM's biowaiver guidance should not be applied in these situations. Similar 
questions may arise if going from the administration of drug in total feed vs. as a top dress.

Sponsor's requests for a biowaiver may be made on the basis either of comparison of formulations or demonstration of solubility. Demonstration of solubility of the active ingredient of Type A medicated articles can be made using the USP definition approach for very soluble, freely soluble or soluble. The minimal requirement (soluble) comprises 'from 10 to 30 parts of solvent need to dissolve one part solute'. An alternative dosage adjusted approach involves determining water solubility, across a defined $\mathrm{pH}$ range, according to the highest expected milligram per kilogram daily intake of drug and the gastric fluid volume of the target animal species. Species specific animal weight and fluid volumes are provided in the guidance notes.

Irrespective of which of the two alternative approaches is selected, the experimental conditions are defined (based on CDER Guidance for Industry). These include the use of buffers of $\mathrm{pH}$ values $1.2,4.6$ and 7.5 , investigated at a temperature of $37 \pm 1{ }^{\circ} \mathrm{C}$ using a minimum of three replicate determinations for each $\mathrm{pH}$, with concentration of drug substance determined using a validated assay.

\section{Canada}

New Guidance for Industry on the Preparation of Veterinary Abbreviated New Drug Submission - Generic Drugs came into effect on April 1, 2010. In some degree the new guidance achieves harmonization with equivalent legislation in the USA. On premixes, there are special requirements for mixing and stability studies. Stability is to be tested for 3 month periods at temperatures of 25 and $40{ }^{\circ} \mathrm{C}$ (at relative humidities of $60 \%$ and $75 \%$, respectively) carried out at the lowest and highest levels of incorporation into feed. The studies also include tests for homogeneous distribution of the premix within the feed, including any tendency to de-mix or electrostatic separation. The sponsor is required to submit a validated analytical method for the drug when mixed in complete feeds at the recommended level and a detailed sampling procedure for analysis of samples is proposed.

If the sponsor requests a waiver from comparative in vivo studies, in vitro data are required to establish pharmaceutical equivalence. The authority 'will consider waiver requests' (not necessarily grant such requests) in the following circumstance: 'If a highly water soluble active ingredient present in a generic feed premix is shown to rapidly dissolve when exposed to a range of physiological $\mathrm{pH}$ values representative of in vivo conditions, it will likely go rapidly into solution when exposed to the fluids in the gastrointestinal tract and effectively behave as an oral solution shortly after administration'. It is not wholly clear from this wording if the ability to dissolve rapidly refers to the premix per se or the finished feed. Alternatively, it may refer to the pure drug substance. The requirements placed on the sponsor in seeking a biowaiver are: '(i) the demonstration of solubility across a pH range of 1.2-7.5 (low, medium and high); and (ii) similar product formulation to ensure that there are no ingredients (including biomass) in the generic formulation that could adversely affect the performance of the generic product by causing a direct pharmacological effect (e.g. altered gastrointestinal transit time, membrane permeability, or drug metabolism) or by inactivating the active ingredient (e.g. chelating agent)'.

Guidance notes within the Canadian document on dissolution testing refer to IRSODs in general and not specifically to premixes, although one assumes from the description outlined in the above paragraph that some premixes, when formulated into the final feed, can be classified as IRSODs. The guidance on dissolution testing indicates that a waiver may be granted for IRSODs, and describes requirements in relation to methodology and apparatus, which should be in accordance with PhEur, BP, USP and CSP pharmacopoeial requirements. It is not clear how these are to be adapted for premixes, but the similarity between test and reference products (in the case of premixes the product is the final feed) should be demonstrated statistically using $\mathrm{f}_{2}$, the similarity factor, which establishes pharmaceutical equivalence. The two dissolution curves (presumably for the final feed containing premix) are considered to be similar if the $f_{2}$ value is $\geq 50$. In order to use mean values, the $\mathrm{CV}$ should not be more than $20 \%$ at early time points and not $>10 \%$ at other time points. On the other hand, if both generic and reference products dissolve $\geq 85 \%$ of label amount in $\leq 15 \mathrm{~min}$ in three dissolution media, the profile comparison using $\mathrm{f}_{2}$ is not necessary. One assumes that, if a generic and pioneer premix does not meet the $\mathrm{f}_{2}$ requirements, then in vivo bioequivalence studies must be undertaken.

\section{European Union}

The current Guidelines for the Conduct of Bioequivalence Studies for Veterinary Medicinal Products (EMEA/CVMP/016/00-CorrFinal) came into force on 11 July 2001. Although they make no mention of premixes, it must be assumed that they encompass them, as they are veterinary medicinal products. However, a new guideline (EMA/CVMP/016/00-Rev.2) has been finalized and will be effect 1 November 2011. This revision states that dosage forms for use in feed may be regarded as IRSODs and be eligible for a biowaiver. They state that premixes and other pharmaceutical forms for use in-feed may be eligible for a biowaiver. Most veterinary medicinal products, excluding suspensions and emulsions, for use in drinking water, milk or milk replacer are likely to be exempted from the demand of in-vivo bioequivalence data.'

The Guideline excludes from granting a biowaiver for premixes containing drugs of low solubility (Class II low solubility, high permeability and Class IV low solubility, low permeability), but allow for the possibility of a biowaiver for Class I (high solubility, high permeability) and Class III (high solubility, low permeability) drugs, even if they contain insoluble constituents as excipients. It is, however, an additional consideration for granting of a biowaiver for Class I compounds that the excipients 'are not expected to have any relevant impact on bioavailability' and for Class III compounds excipients have to be 'qualitatively the same and quantitatively very similar' for pioneer and generic products. In practice, the latter criterion is 
no small distinction, because it would generally not be the case that generic excipients are qualitatively the same and quantitatively very similar to those of the pioneer product. This distinction between Class I and Class III compounds, with respect to eligibility for a biowaiver, is justified in the Guideline by the view that 'the risks of an inappropriate biowaiver decision should be more critically reviewed (e.g. site-specific absorption, risk for transport protein interactions at the absorption site, excipient composition and therapeutic risks) for products containing BCS Class III than for Class I active substances'. Even for Class I drugs, however, the impact of excipients though considered to be 'rather unlikely, it cannot be completely excluded...therefore it is advisable to use similar amounts of the same excipients' in test and reference products.

From the above EMA Guidance on both actives and excipients, it would seem likely that generic premixes containing actives of Classes II and IV would always be required to establish bioequivalence in vivo and this would also apply to some premixes in Classes I and III. It is also noted that the BCS classification will be species specific, because interspecies differences in diet and gastrointestinal tract physiology might result in differences in the absorption characteristics of various compounds. Nevertheless, for Class I and Class III compounds a biowaiver could, in principle, be granted provided dissolution criteria were met. The criteria set for IRSODs of Classes I and III comprise: (i) more than $85 \%$ in vitro dissolution of test and reference product within $15 \mathrm{~min}$; and (ii) complete dissolution in a volume of buffer corresponding to the gastric volume of the target species of at least three buffers in the range of $\mathrm{pH}$ values of $1.0-7.5$ at $37^{\circ} \mathrm{C}$. Additional dissolution data may be required at $\mathrm{pH}$ values in which the active substance has minimum solubility, and the use of any surfactant is strictly discouraged. These in vitro studies have been set for IRSODs in general. The draft Guideline makes no mention of whether or how they should be adapted for premixes, but simply state that the methodology should be in accordance with pharmacopoeial requirements. It is, however, stated that 'alternative methods can be considered when justified'. In light of the discussion above, in relation to FDA Guidelines on biowaivers, the question arises as to whether drug substance solubility, across a range of $\mathrm{pH}$ values, does suffice to generally to justify a biowaiver. Pre-mixes are complex formulations and, as suggested by del Castillo and Wolff (2006), the dissolution rate of the drug product may be important, at least for some instances, even when the drug substance is classified as water soluble.

\section{Australia}

A new draft Guideline on Bioequivalence and Generic Equivalence has been issued by the Australian Regulatory Authority (APVMA) and is currently under review. Section 4 describes product classes, which are regarded as closely similar to a registered reference product and require assessment of chemistry and manufacture data only. Such generic products are registered under Category 6, which means that neither in vivo bioequivalence nor in vitro dissolution tests are required. Close similarity for a generic product is accepted, if the products are pharmaceutically equivalent and the use pattern (including target species, dose rates, route of administration, withholding periods) label claims and label instructions are the same as the reference product (except there can be fewer or reduced claims than for the reference product).

Medicated premix products in powder or granular dose forms are classified as low formulation dependent dosage forms, under the sub-heading, 'dose forms for administration in or with feed where the only non-active constituents are cereal/vegetable'. The draft guideline states 'bioequivalence studies are not relevant for medicated premix products in powder or granular dose forms, intended for administration in animal feed. If the carrier constituent is not cereal-based and is different to that of the reference product and/or is present in a concentration that has the potential to affect the occupational health and safety of human handlers of the product, this will be outside the scope of a pharmaceutical equivalence consideration'.

\section{CONFLICTS OF INTEREST}

R.P.H is employed by Elanco Animal Health which is a for-profit animal health company; P.L., P.-L.T. declare no conflicts of interest; D.C. has not declared any conflicts of interest.

\section{REFERENCES}

Ali, D.N. \& Hennessy, D.R. (1996) The effect of level of feed intake on the pharmacokinetic disposition and efficacy of ivermectin in sheep. Journal of Veterinary Pharmacology \& Therapeutics, 19, 89-94.

Amidon, G.L., Lennernas, H., Shah, V.P. \& Crison, J.R. (1995) A theoretical basis for a biopharmaceutic drug classification: the correlation of in vitro drug product dissolution and in vivo bioavailability. Pharmaceutical Research, 12, 413-420.

Benet, L.Z. (2009) Predicting drug disposition via application of a biopharmaceutics drug disposition classification system. Basic \& Clinical Pharmacology \& Toxicology, 106, 162-167.

Benet, L.Z., Amidon, G.L., Barends, D.M., Lennernäs, H., Polli, J.E., Shah, V.P., Stavchansky, S.A. \& Yu, L.X. (2008) The use of BDDCS in classifying the permeability of marketed drugs. Pharmaceutical Research, 52, 483-488.

Blume, H.H. \& Schug, B.S. (1999) The biopharmaceutics classification system (BCS): class III drugs - better candidates for BA/BE waiver? European Journal of Pharmaceutical Sciences, 9, 117-121.

Burton, P.S., Goodwin, J.T., Vidmar, T.J. \& Amore, B.M. (2002) Predicting drug absorption: how nature made it a difficult problem. Journal of Pharmacology and Experimental Therapeutics, 303, 889-895.

del Castillo, J.R.E. \& Wolff, T. (2006) Therapeutic lung exposure to feedadministered chlortetracycline is premix brand dependent. Proceedings/American Association of Swine Veterinarians, 143-148.

Davit, B.M., Conner, D.P., Fabian-Fritsch, B., Haidar, S.H., Jiang, X., Patel, D.T., Seo, P.R., Suh, K., Thompson, C.L. \& Yu, L.X. (2008) Highly variable drugs: observations from bioequivalence data submitted to the FDA for new generic drug applications. American Association of Pharmaceutical Scientists Journal, 10, 148-156.

Haidar, S.H., Davit, B., Chen, M.L., Conner, D., Lee, L.M., Li, Q.H., Lionberger, R., Makhlouf, F., Patel, D., Schuirmann, D.J. \& Yu, L.X. 
(2008a) Bioequivalence approaches for highly variable drugs and drug products. Pharmaceutical Research, 25, 237-241.

Haidar, S.H., Makhlouf, F., Schuirmann, D.J., Hyslop, T., Davit, B., Conner, D. \& Yu, L.X. (2008b) Evaluation of scaling approach for the bioequivalence of highly variable drugs. American Association of Pharmaceutical Scientists Journal, 10, 450-454.

Kortejärvi, H., Urtti, A. \& Yliperttula, M. (2007) Pharmacokinetic simulation of biowaiver criteria: the effects of gastric emptying, dissolution, absorption and elimination rates. European Journal of Pharmaceutical Sciences, 30, 155-166.

Lees, P., Taylor, J.B.O., Higgins, A.J. \& Sedgwick, A.D. (1988) In vitro and in vivo studies on the binding of phenylbutazone and related drugs to equine feeds and digesta. Research in Veterinary Science, 44, 50-56.

Maitho, T.E., Lees, P. \& Taylor, J.B. (1986) Absorption and pharmacokinetics of phenylbutazone in Welsh Mountain ponies. Journal of Veterinary Pharmacology and Therapeutics, 9, 26-39.

Martinez, M.N. \& Hunter, R.P. (2010) Current challenges facing the determination of product bioequivalence in veterinary medicine. Journal of Veterinary Pharmacology and Therapeutics, 33, 418-433.

Martinez, M.N., Langston, C., Martin, T. \& Conrnes, D. (2002a) Challenges associated with the evaluation of veterinary product bioequivalence: an AAVPT perspective. Journal of Veterinary Pharmacology and Therapeutics, 25, 201-220.

Martinez, M., Augsburger, L., Johnston, T. \& Jones, W.W. (2002b) Applying the biopharmaceutics classification system to veterinary pharmaceutical products. Part I: biopharmaceutics and formulation considerations. Advanced Drug Delivery Reviews, 54, 805-824.

Martinez, M., Amidon, G., Clarke, L., Jones, W.W., Mitra, A. \& Riverie, J. (2002c) Applying the biopharmaceutics classification system to veterinary pharmaceutical products. Part II. Physiological considerations. Advanced Drug Delivery Reviews, 54, 825-850.

Neuhofel, A.L., Wilton, J.H., Victory, J.M., Hejmanowski, L.G. \& Amsden, G.W. (2002) Lack of bioequivalence of ciprofloxacin when administered with calcium-fortified orange juice: a new twist on an old interaction. Journal of Clinical Pharmacology, 42, 461-466.

Polli, J.E., Abrahamsson, B.S., Yu, L.X., Amidon, G.L., Baldoni, J.M., Cook, J.A., Fackler, P., Hartauer, K., Johnston, G., Krill, S.L., Lipper,
R.A., Malick, W.A., Shah, V.P., Sun, D., Winkle, H.N., Wu, Y. \& Zhang, H. (2008) Summary workshop report: bioequivalence, biopharmaceutics classification system and beyond. American Association of Pharmaceutical Scientists Journal, 10, 372-379.

Ramirez, E., Laosa, O., Guerra, P., Duque, B., Mosquera, B., Borobia, A.M., Lei, S.H., Carcas, A.J. \& Frias, J. (2010) Acceptability and characteristics of 124 human bioequivalence studies with active substances classified according to the biopharmaceutic classification system. British Journal of Clinical Pharmacology, 70, 694-702.

Schug, B.S., Brendel, E., Wolf, D., Wonnemann, M., Wargenau, M. \& Blume, H.H. (2002a) Formulation-dependent food effects demonstrated for nifedipine modified-release preparations marketed in the European Union. European Journal of Pharmaceutical Sciences, 15, 279-285.

Schug, B.S., Brendel, E., Wonnemann, M., Wolf, D., Wargenau, M., Dingler, A. \& Blumme, H.H. (2002b) Dosage form-related food interaction observed in a marketed once-daily nifedipine formulation after a high-fat American breakfast. European Journal of Clinical Pharmacology, 58, 119-125.

Shryock, T.R., Klink, P.R., Readnour, R.S. \& Tonkinson, L.V. (1994) Effect of bentonite incorporated in a feed ration with tilmicosin in the prevention of induced Mycoplasma gallisepticum airsacculitis in broiler chickens. Avian Disease, 38, 501-505.

Toutain, P.L. \& Bousquet-Melou, A. (2004) Bioavailability and its assessment. Journal of Veterinary Pharmacology and Therapeutics, 27, 455-466.

Van Duijkeren, E., Kessels, B.G.F., Sloet van Oldruitenborgh-Oosterbaan, M.M., Breukink, H.J., Vulto, A.G. \& Van Miert, A.S.J.P.A.M. (1996) In vitro and in vivo binding of trimethoprim and sulphachlorpyridazine to equine food and digesta and their stability in caecal contents. Journal of Veterinary Pharmacology and Therapeutics, 19, 281-287.

Welsh, J.C.M., Lees, P., Stodulski, G., Cambridge, H. \& Foster, A.P. (1992) Influence of feeding schedule on the absorption of orally administered flunixin in the horse. Equine Veterinary Journal, 24 (Suppl. 11), 62-65.

Yu, L.X., Amidon, G.L., Polli, J.E., Zhao, H., Mehta, M.U., Conner, D.P., Shah, V.P., Lesko, L.J., Chen, M.L., Lee, V.H.L. \& Hussain, A.S. (2002) Biopharmaceutics classification system: the scientific basis for biowaiver extensions. Pharmaceutical Research, 19, 921-925. 